\title{
Endovascular management of the post-traumatic common carotid artery pseudoaneurysms
}

\author{
Pankaj Jariwala ${ }^{A, B, C, D, E, F}$, Arshad Punjani ${ }^{B, D}$, Rajeev Kamble ${ }^{D}$ \\ Yashoda Hospitals, Somajiguda, Hyderabad, 500082, India
}

\section{Abstract}

\begin{abstract}
Purpose: Pseudoaneurysms of the common carotid artery secondary to high-velocity fragment injuries to the head and neck in patients are uncommon lesions. Multi-detector computed tomography angiography should be performed on all patients suffering from high-speed fragment injuries of the head and neck.
\end{abstract}

Case presentation: We share our experience with the endovascular management approach for the closure of 2 separate pseudoaneurysms involving the left common carotid artery.

Conclusions: Numerous options for surgical and endovascular treatment of these lesions are available. Endovascular therapy in patients with common carotid artery pseudoaneurysms offers a reliable and non-invasive management option. It helps in the mitigation of its rupture and thromboembolic complications.

Key words: pseudoaneurysm, carotid artery, covered stent, traumatic neck injuries.

\section{Introduction}

Pseudoaneurysm (PSA) is defined as a pulsating encapsulated haematoma that develops in connection with the lumen of a ruptured vessel after an injury to all layers of the arterial wall. The lack of a 3-layer structure distinguishes it from a true aneurysm, and it makes sudden rupture more probable. The incidence of common carotid artery PSAs (CCA-PSA) of all extracranial aneurysms is $0.1-0.3 \%$ [1,2].

The aim of treating CCA-PSA is to avoid rupture, embolic complications, and neurological disability associated with the procedure. The treatment choices include surgery and endovascular therapy [3]. Traditionally, surgery was the treatment of choice, but due to improved equipment availability, the new endovascular techniques provided a less invasive solution with increased efficacy.

\section{Case presentation}

A 45-year-old-male labourer presented with a steadily growing pulsatile mass on the left side of his neck, which had been present for 2 months. On detailed retrospective interrogation, he gave a history of a high-speed shrapnel injury while working at a construction site. Following this, he experienced a minor injury to the neck that healed with the help of first aid treatment. Physical examination showed a painless, pulsatile mass on the left neck region. The neurological test was normal and his vital signs stable.

The multi-detector computed tomography (MDCT) scan revealed the presence of 2 different PSAs, one larger in size and fusiform in shape, and the other smaller sized, oval-shaped from the posterolateral and anteromedial walls of the common carotid artery, respectively (Figure 1). A selective carotid angiogram showed 2 PSAs as described earlier from the distal segment of left CCA below the bifurcation with an appropriate landing zone (Figure 2).

The endovascular intervention of the PSA first involved the placement of a 0.035 -inch guidewire (Terumo Corporation, Japan) via the femoral approach into the left internal carotid artery across the left CCA using an $8 \mathrm{~F}$ long-sheath. We decided to treat the patient with a stent graft [covered stent]; hence, a $25 \times 7 \mathrm{~mm}$ Viabahn (Gore

\section{Correspondence address:}

Dr. Pankaj Jariwala, Yashoda Hospitals, Somajiguda, Hyderabad, 500082, India, e-mail: pankaj_jariwala@hotmail.com

Authors' contribution:

A Study design · B Data collection · C Statistical analysis · D Data interpretation · E Manuscript preparation · F Literature search · G Funds collection 

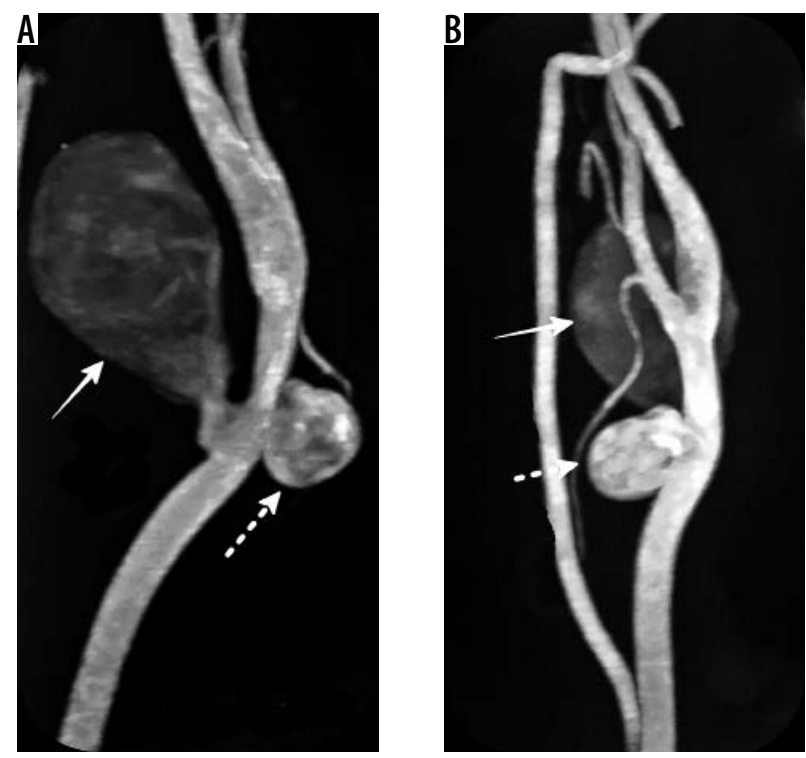

Figure 1. Multidetector computed tomography angiography of the left carotid artery. Maximum intensity projection images in sagittal section (A) and coronal section (B) showing 2 separate pseudoaneurysms, a largesized, fusiform-shaped (solid white arrow) along the posterolateral wall and another small-sized, oval-shaped along the anteromedial wall (dashed white arrow) arising at the same level from the distal segment of the left common carotid artery below the bifurcation

and associates, USA) self-expanding covered stent was then expanded in the CCA across both of the PSAs leading to their complete closure (Figures 3 and 4). The patient was discharged with dual antiplatelet therapy (aspirin $150 \mathrm{mg}$ and clopidogrel $75 \mathrm{mg}$ ) on the $2^{\text {nd }}$ day post procedure. Patients had 3, 6, and 12 months of clinical followup, and each time carotid Doppler was performed, showing a patent stent without PSA recurrence or endoleak. Thereafter, he was recommended for an annual check-up.

\section{Discussion}

Traumatic CCA-PSAs, although rare, can be life-threatening and difficult to treat. Blunt, penetrating, or iatrogenic injuries may result in traumatic extracranial carotid PSAs. Penetrating carotid artery injuries secondary to high-velocity fragment injuries occur in up to $10 \%$ of all neck trauma cases and carry a high mortality rate of up to $50 \%$ if remains unmanaged $[2,4]$.

MDCT has been used as a diagnostic imaging modality in most institutions because it is non-invasive, widely available, and has high sensitivity and specificity. Biffl et al. suggested a method of grading these traumatic neck injuries. The PSAs were considered as grade III lesions as they arise from full-thickness wall damage, resulting in a localized partially perfused haematoma [5].

Management of extracranial carotid lesions depends on the extent of the lesion and the overall general condition of the patient, from surgical treatment like open repair to endovascular treatment like deployment of a covered stent. Because of the reduced invasiveness and improved procedural effectiveness, endovascular repair has received more acceptance [6]. The approaches for endovascular management rely on the age of the patient, the location of PSA, and other associated diseases of the cerebral circulation and the status of its collateral circulation. The location of the PSA, whether extra- or intra-cranial, affects the option of which hardware to use. Extracranial CCA is a simple anatomical structure, without any major branches [3].

Many previously described methods like coiling or stent-assisted coil embolization may not be successful if the source of bleeding is not addressed. The use of the covered stent remains the treatment of choice because
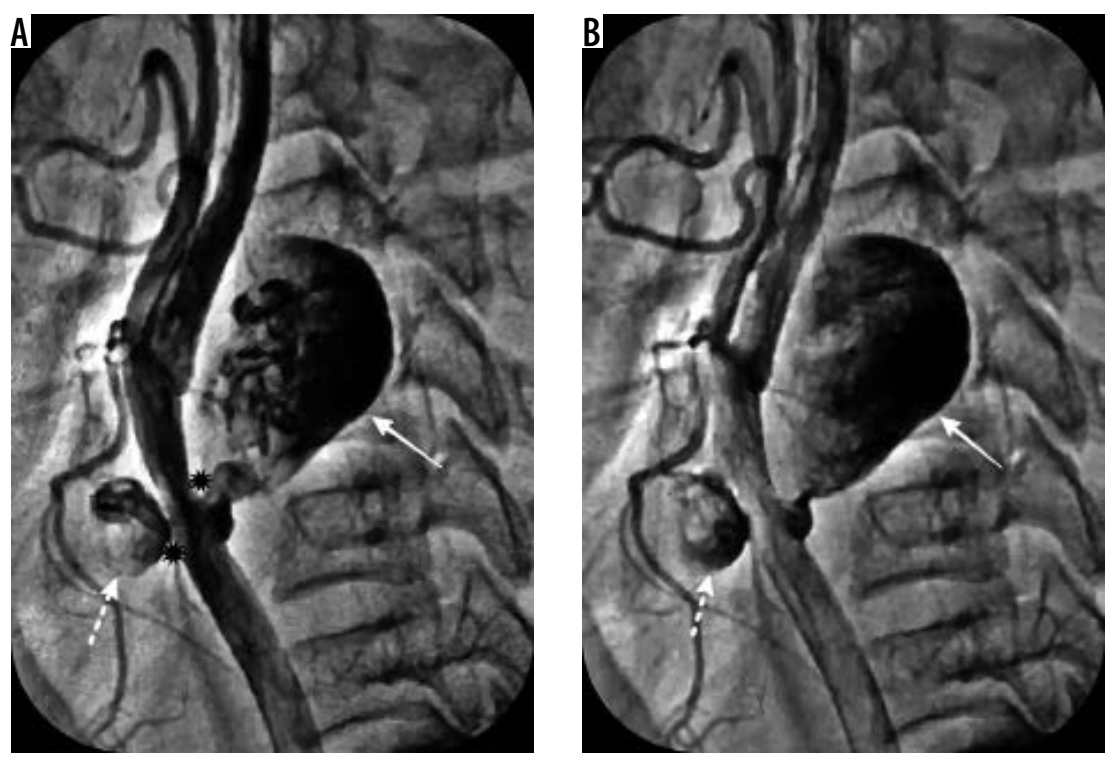

Figure 2. Selective angiography of the left common carotid artery. Pseudoaneurysms showing in 2 different phases of filling, (A) partially and (B) near-complete filling phases due to slow, phasic filling secondary to their narrow necks (black asterisks; a). It demonstrated 2 separate pseudoaneurysms: a largesized, fusiform (solid white arrow) and another small-sized, oval-shaped (dashed white arrow) arising at the same level from the left common carotid artery below the bifurcation 


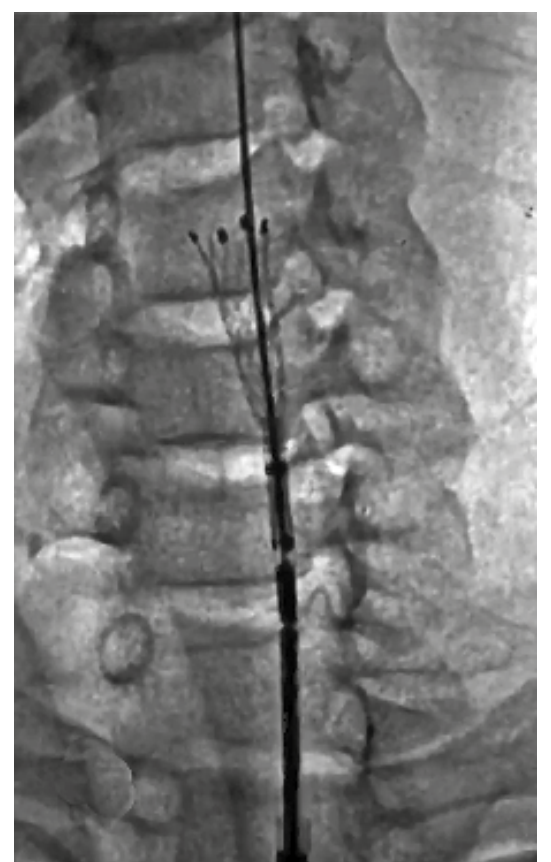

Figure 3. Cine fluoroscopy of the left neck region. A partially expanded self-expanding covered stent seen across the distal segment of the left common carotid artery

it maintains the patency of the parent artery and results in early complete obliteration of the PSA [7-9]. Both the balloon and self-expanding covered stents available. It is now well established that balloon-expandable stents are not suitable for extracranial carotid arteries because they are subjected to increased external compression, which leads to serious damage and its occlusion [10].

Our option was to use a self-expanding, covered stent, which that is more durable and less likely to be compromised by external stress. For this reason, the Viabahn stent (Gore \& Associates), which is a polytetrafluoroethylene (PTFE)-covered nitinol self-expanding stent, is well suited because the PTFE is nonporous and prevents type IV endoleak $[2,11]$. Hoppe et al. reported on their experience with carotid artery stent-grafts in 25 patients. Their rate of technical success was $100 \%$. Procedural dissection and short-term complications were observed, respectively, in $7.4 \%$ and $11 \%$ of their patients without any long-term medical complications [12].

The post-procedure antithrombotic strategy is critical for long-term effectiveness, because the probability of stent occlusion in this setting has been documented to be much higher compared to atherosclerotic disease. For this reason, despite the high occurrence, no particular protocol was established, and the strategy of dual antiplatelet therapy with clopidogrel, and aspirin was recommended based on the experience [2,13]. Regular follow-up of the patient should be carried out using clinical examination and carotid Doppler at an interval of 3, 6, and 12 months initially and later annually. There is no clear established

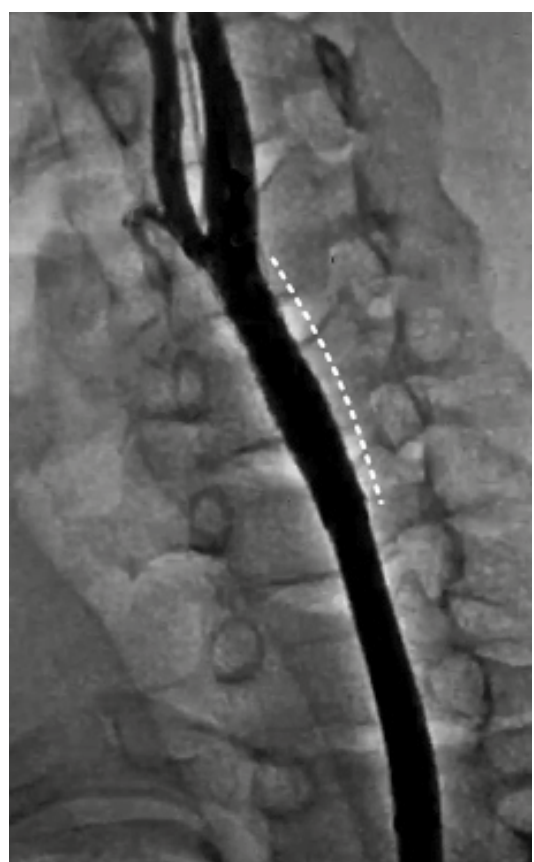

Figure 4. Conventional angiography of the left carotid artery. Deployment of the covered stent (curved white dashed line) leading to the complete closure of both of the pseudoaneurysms with the normal flow in the left carotid system

protocol, and clinicians should use their experience based on their institutional practices.

\section{Conclusions}

Carotid artery injury associated with high-velocity fragment trauma to the head and neck is a serious condition, but early diagnosis and successful treatment results in low morbidity and mortality. The effect of undisclosed lesions can be serious, and every effort should be made to screen all high-risk patients if there is any history of neck injury in the presence of neck swelling. Endovascular interventions offer a safe and reliable method of treatment for CCA-PSAs with the advent of improved hardware and skills; furthermore, management must be customized based on clinical and imaging profiles for each patient such as aneurysm location, characteristics, etc. Unfortunately, there are no clear guidelines in the current literature to suggest the appropriate plan of treatment due to its low incidence, limitations in the documentation of outcomes, and the inconsistency of indication. To specify the treatment options for CCA-PSA, a multicentre international registry is required. Also, postprocedural dual antiplatelet treatment and follow-up using ultrasound are crucial to increasing the likelihood of successful longterm outcomes.

\section{Conflict of interest}

The authors report no conflict of interest. 


\section{References}

1. Gupta K, Dougherty K, Hermman H, Krajcer Z. Endovascular repair of a giant carotid pseudoaneurysm with the use of viabahn stent graft. Catheter Cardiovasc Interv 2004; 62: 64-68.

2. Yamada R, Miller C, Guimaraes M, Schonholz C. Overview of endovascular management of traumatic carotid lesions. Endovasc Today 2016; 15: 79-82.

3. Kumar A, Prabhakar A, Gupta V, et al. Endovascular management of internal carotid artery pseudoaneurysms: a single-center experience of 20 patients. Neurol India 2018; 66: 1067-1074.

4. Tisherman SA, Bokhari F, Collier B, et al. Clinical practice guideline: penetrating zone II neck trauma. J Trauma 2008; 64: 1392-1405.

5. Biffl WL, Moore EE, Offner PJ, et al. Blunt carotid arterial injuries: implications of a new grading scale. J Trauma 1999; 47: 845-853.

6. Gupta V, Niranjan K, Rawat L, Gupta AK. Stent-graft repair of a large cervical internal carotid artery pseudoaneurysm causing dysphagia. Cardiovasc Intervent Radiol 2009; 32: 558-562.

7. Koyanagi M, Nishi S, Hattori I, Horikawa F, Iwasaki K. Stentsupported coil embolization for carotid artery pseudoaneurysm as a complication of the endovascular surgery - case report. Neurol Med Chir (Tokyo) 2004; 44: 544-547.
8. Tokunaga K, Kusaka N, Nakashima H, Date I, Ohmoto T. Coil embolization of intradural pseudoaneurysms caused by arterial injury during surgery: report of two cases. AJNR Am J Neuroradiol 2001; 22: 35-39.

9. Pai B, Limaye U, Varma R. Endovascular management of a refractory traumatic aneurysm of the internal carotid artery using a covered stent graft. Neurol India 2008; 56: 488-490.

10. Schönholz C, Krajcer Z, Parodi JC, et al. Stent-graft treatment of pseudoaneurysms and arteriovenous fistulae in the carotid artery. Vascular 2006; 14: 123-129.

11. Maras D, Lioupis C, Magoufis G, et al. Covered stent-graft treatment of traumatic internal carotid artery pseudoaneurysms: a review. Cardiovasc Intervent Radiol 2006; 29: 958-968.

12. Hoppe H, Barnwell SL, Nesbit GM, Petersen BD. Stent-grafts in the treatment of emergent or urgent carotid artery disease: review of 25 cases. J Vasc Interv Radiol 2008; 19: 31-41.

13. Cothren CC, Moore EE, Ray CE Jr, et al. Carotid artery stents for blunt cerebrovascular injury: risks exceed benefits. Arch Surg 2005; 140: 480-485. 